\title{
Cross-layer modeling of capacity of UMTS/HSDPA networks under dynamic user setting
}

\author{
Tijani Chahed, Mariana Dirani \\ UMR CNRS 5157 \\ GET/Institut National des Télécommunications \\ 9 rue C. Fourier - 91011 Evry CEDEX - France \\ \{tijani.chahed, mariana.dirani\}@int-evry.fr
}

\begin{abstract}
We focus in this paper on the cross-layer modeling of the integration of streaming and elastic flows in the downlink of UMTS/HSDPA systems. Streaming flows are transported over dedicated channels whereas elastic ones shall share the left over capacity according to opportunistic scheduling implemented at the MAC layer. We first study the system at the MAC layer and then make use of these results at the flow level where the system is studied in a dynamic setting. The quasi-stationary assumption makes it possible to obtain a product form expression for the steady-state probabilities of the system. Flow level dynamics are then used at the packet level to investigate TCP performance and quantify the probability of a spurious event, namely timeout. Several performance metrics are then derived and give hints into dimensioning issues of such a system.
\end{abstract}

\section{INTRODUCTION}

Third generation mobile systems are currently witnessing the arrival of elastic data flows over the downlink shared channels (DSCHs), along with classical streaming flows transported over dedicated channels (DCHs). The actual implementation of DSCH is based on High Speed Downlink Packet Access (HSDPA) standard in UMTS, or equivalently High Data Rate (HDR) in equivalent CDMA2000 1xEV-DO systems.

Both techniques bring control closer to the User equipment (UE) at the Node B so as to be reactive on even smaller time scales, so called Transport Time Interval (TTI), as low as 2 mseconds. They deploy sophisticated adaptive modulated technique (AMC) and make use of Hybrid ARQ (HARQ) to fight against corruption of frames. They rely on opportunistic type of scheduling to allocate radio resources to competing data users whereby users experiencing best radio conditions are granted access to resources, without sacrificing to considerations of fairness. Proportional Fair SCheduling (PFS) is one such scheduling mechanism often envisaged in this context [1].

The main challenge in the modeling of the simultaneous presence of both types of traffic, streaming and elastic, resides in the fact that streaming flows have a duration that is independent of the state of the system. Whereas TCP-based elastic ones shall share the left over capacity fairly among themselves and have thus a service duration closely related to the system capacity and the number of users sharing it.

This sharing follows in fact an opportunistic scheduling scheme wherein a trade-off is to be assured between granting resources to users experiencing the best radio conditions and fairness, in terms of time essentially. We thus focus at first on the MAC layer where this scheduling is implemented and investigate the impact of Proportional Fair Scheduling (PFS) on the elastic flows, in comparison with other scheduling mechanisms such as Round Robin (RR) or Max C over I, an algorithm that grants resources to users with best radio conditions with no concern about fairness.

We next focus at the flow level and develop an exact Markovian analysis as well as an approximate one based on the quasi-stationary hypothesis. The quasi-stationary assumption tells us that, assuming voice flows have quite slow dynamics, both in their arrival and departure, it is possible to identify some stationary regions during which the sharing of resources between elastic flows can be studied for a fixed shared capacity figure [2] and is obtained in a cross-layer fashion from the MAC layer.

We eventually investigate TCP dynamics at the packet level, for a given number of flows obtained in a cross-layer fashion from the flow level, and quantify the probability of a spurious event, namely time-out, due in this case to an excessive delay in the wireless portion of the end-to-end system which may be wrongly interpreted by the source as an indication of loss.

The remainder of this work is organized as follows. Section II briefly illustrates the end-to-end system. Section III details our model, including modeling of HSDPA dynamics at the MAC layer, the system dynamics at the flow level and TCP dynamics at the packet level. Section IV contains our analysis for the underlying Markov Chain. Section V shows some numerical results comparing our analysis to the exact one. Section VI concludes the paper.

\section{END-TO-END SYSTEM}

Our reference model is as shown in Figure 1 which shows for the case of data flows, and following the direction of the downlink, an (FTP) server, a (wireline) Internet cloud, the UMTS Core Network (CN), the Radio Network Controller (RNC), the base station and the User Equipment (UE). Unlike standard UMTS, HSDPA puts intelligence and control mostly in the base station and not the RNC. On the other hand, voice flows are managed through the RNC over dedicated links. 


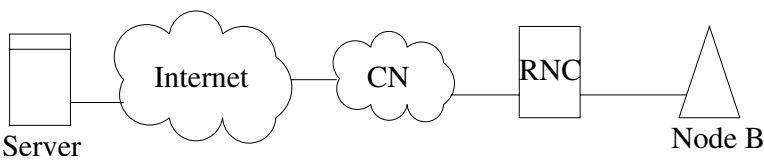

Fig. 1. End-to-end system model

\section{MODEL}

\section{A. Modeling HSDPA dynamics at the MAC layer}

At the MAC layer, HSDPA is managed through PFS which works as follows. Let $r_{i}(t)$ denote the instantaneous data rate at which the ith user can transmit. It depends on the distance $d(i)$ between the base station and user $i$ location in the cell, which represents path loss, as well as the radio conditions $S(i)$ that the user experiences, which represents fast fading and depends on parameters such as speed. hence,

$$
r_{i}=C_{s h} d(i) S(i)
$$

where $S$ is a random variable representing the fast fading component of the system. In the case of Rayleigh fading channel, the distribution of the envelope for the channel response at any time (assuming uniformly distributed phase in the interval $(0,2 \pi))$ is given by

$$
\frac{2 s}{\Omega} e^{-\frac{s^{2}}{\Omega}}
$$

where $\Omega=E\left(S^{2}\right)$ [3]. $d($.$) is given by$

$$
\left(\frac{d_{0}}{d}\right)^{a}
$$

for all $d>d_{0}$ and is equal to 1 otherwise. $a$ is the path loss exponent (typical values are between 2 and 5) [4].

Let $R_{i}(t)$ be the average data rate for user $i$. In slot $t$, the user $i^{*}$ with the largest $\frac{r_{i}(t)}{R_{i}(t)}$ ratio is the one who is granted all (shared) system capacity $C_{s h}$ and transmits then at rate $r_{i}(t)$.

The average throughput $R_{i}(t)$ is then updated using an exponentially weighted low-pass filter [1]

$$
R_{i}(t+1)=\left(1-\frac{1}{\tau}\right) R_{i}(t)+\frac{1}{\tau} r_{i}^{*}(t)
$$

for user $i^{*}$ and

$$
R_{i}(t+1)=\left(1-\frac{1}{\tau}\right) R_{i}(t)
$$

for all other users. Or,

$$
R_{i}(t+1)=\left(1-\frac{1}{\tau}\right) R_{i}(t)+\frac{1}{\tau} r_{i}(t) \arg \max _{1, \ldots, N}\left\{\frac{r_{i}(t)}{R_{i}(t)}\right\}
$$

where $\tau$ is the time interval over which the past is activity of the scheduler versus present granting is weighted.

Now,

$$
\mu_{i}=\lim _{t \rightarrow \infty} R_{i}(t)
$$

To find $\mu^{d}$, we need to average out the previous value over all users in progress in the cell. So,

$$
\mu^{d}=\frac{1}{n^{d}} \sum_{i=1}^{n^{d}} \mu_{i}
$$

This term is now to be used at the flow level, as shown next.

\section{B. Modeling system dynamics at the flow level}

Let us now consider the system capacity at the flow level in a dynamic setting, i.e., with flows of the two types arriving to the system and departing from it.

To model the presence of both streaming and elastic flows in the downlink of UMTS/HSDPA systems, let us consider the following Markov Chain. Let the state be defined by the number of users of both types, streaming and data, $n^{v}$ and $n^{d}$, respectively. Arrival of both types of flows follows a Poisson process with mean rates $\lambda^{v}$ and $\lambda^{d}$, respectively. Streaming calls are assumed to be of constant bit rates and served over dedicated channels for a mean duration of time equal to $\bar{s}$ units of time, and hence a mean (exponentially distributed) service rate $\mu^{v}=1 / \bar{s}$. Data calls are however multiplexed over HSDPA-shared channels with mean service rate equal to $\mu^{d}$. The latter is function of the (variable) capacity $C_{s h}$ of the shared link, the opportunistic scheduling that governs its sharing as well as the number of users sharing it, $n^{d}$.

Remark 1. It is possible to intoduce several classes of data calls, in terms of distance of the UE to the base station as well as the radio conditions experienced by it. We simply explicit the number of users of each class in the above mentioned Markov Chain instead of the sole $n^{d}$ term.

Note that the numbers of flows of each type, $n^{v}$ and $n^{d}$ are constrained by the total power $P$ at the base station and corresponding capacity $C$ as follows. For power control to be feasible, we have

$$
n^{v} \mu^{v}+C_{s h} \leq C
$$

where $C_{s h}=n^{d} \mu^{d}$.

Remark 2. $C_{s h}$ is the leftover capacity after voice has been served. Specific CAC policy may impose lower limit on $C_{s h}$ as well as on the maximum number $n_{\max }^{d}$ of data users sharing it.

1) Exact analysis: Back to our Markov Chain. Consider the stochastic process $N(t)=\left(n^{v}(t), n^{d}(t)\right)$. It can be shown that $N(t)$ is a Continuous Time Markov Chain (CTMC), with flow state diagram as shown in Figure 2.

This process is a quasi birth-death process. The infinitesimal generator $Q$ of this chain is given in the next page where $V$ is a tri-diagonal matrix given by

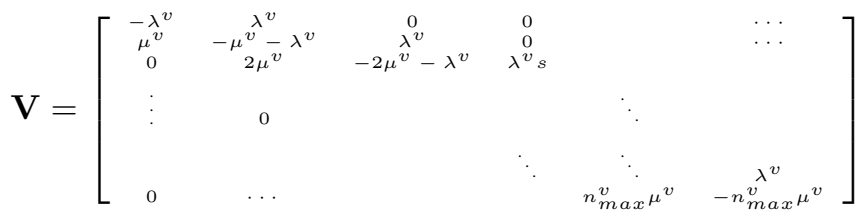




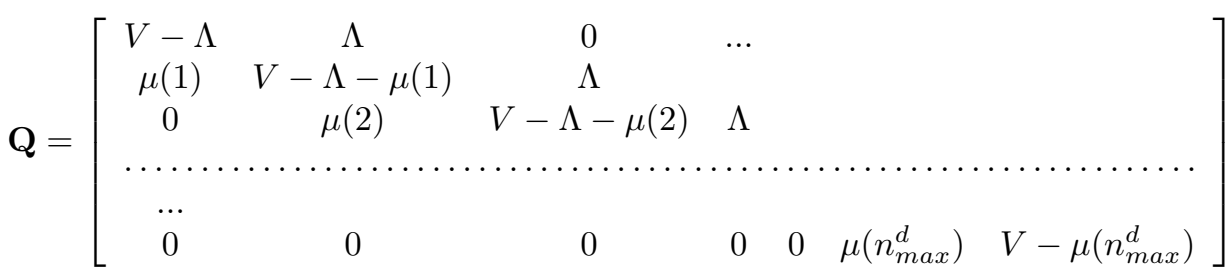

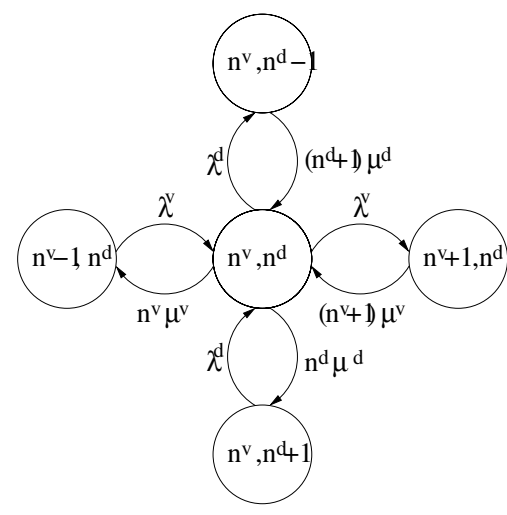

Fig. 2. Markov chain representing the users in the system.

and $\Lambda$ is a diagonal matrix representing the arrivals of new file transfers.

The solution $\Pi$ is then simply obtained through solving the set of linear equations $\Pi Q=0$. The latter are not all linearly independent and so we should also add the one that equates the sum of all probabilities to 1 .

2) Quasi-stationary approximation: Let us now turn to the evaluation of the system performance under the quasistationary approximation. The latter assumes that the voice traffic dynamics, especially arrivals, are much slower than the elastic one. We are hence able to study the sharing of elastic ones, in the framework of M/G/1 queue - Processor Sharing (PS), at time intervals where capacity is indeed constant.

Let $\bar{n}^{d}$ denote the mean number of data flows in the system. In the absence of any admission control, this number can go to infinity. It is given by

$$
\bar{n}^{d}=\sum_{k=0}^{\infty} k \operatorname{Pr}\left(n^{d}=k\right)
$$

Now,

$$
\operatorname{Pr}\left(n^{d}=k\right)=\sum_{j} \operatorname{Pr}\left(n^{d}=k \mid n^{v}=j\right) \operatorname{Pr}\left(n^{v}=j\right)
$$

and

$$
\operatorname{Pr}\left(n^{d}=k \mid n^{v}=j\right)=\frac{1}{G} \Pi_{i=1}^{k} \frac{\lambda^{d} E[V]}{\mu^{d}\left(C-j \mu^{v}, i\right)}
$$

where $G$ is the normalizing constant and $E(V)$ is the mean file size. Moreover,

$$
\operatorname{Pr}\left(n^{v}=j\right)=\operatorname{Pr}\left(n^{v}=0\right) \Pi_{k=0}^{j-1} \frac{\lambda^{v}}{(k+1) \mu^{v}}
$$

for all $j \leq n_{\max }^{v}$ and corresponding to an $\mathrm{M} / \mathrm{M} / \mathrm{m} / \mathrm{m}$ queue. $\operatorname{Pr}\left(n^{v}=0\right)$ is found through the condition $\sum_{i} \operatorname{Pr}\left(n^{v}=\right.$ $i)=1$. $n_{\max }^{v}$ is the maximum number of voice calls that can be accommodate when no data calls are in progress in the system. For $d^{v}$ denoting the (constant) bit rate per dedicated link, $n_{\max }^{v}=C / d^{v}$.

3) Performance measures: The mean transfer time of a data flow is then given by Little's theorem as

$$
T=\frac{\overline{n^{d}}}{\lambda^{d}\left(1-B^{d}\right)}
$$

where $B^{d}$ is the blocking probability, if any, of data calls.

These blocking probabilities, of both types of flows, are equal to the probabilities of the saturation states, $\sum_{i} \pi\left(n_{\max }^{v}, i\right)$ and $\sum_{i} \pi\left(i, n_{\max }^{d}\right)$, respectively.

\section{Modeling TCP dynamics at the packet level}

So far, we assumed a perfect and immediate sharing of resources between TCP flows at the flow level. Let us now investigate to which degree this assumption is true.

At the packet level, the problem can be formulated as a fixed point one, which means that the throughput of a TCP flow depends on the probability of loss and the probability of loss depends on the throughput. The convergence of the fixed-point process is obtained iteratively.

Consider the buffer at the base station which we consider of finite length $K$. It can be modeled by an M/G/1/K queue with vacations where vacations denote the time during which the server is busy serving someone else. The input rate $\lambda$ obeys to TCP and is equal to [5]

$$
\lambda=\frac{1}{(R T T+W) \sqrt{\frac{2 b p}{3}}+T_{0} \min \left(1,3 \sqrt{\frac{3 b p}{8}}\right) p\left(1+32 p^{2}\right)}
$$

where RTT is the round trip time excluding the time spent in the buffer at the base station, $b$ is the number of packets ACKed in one cumulative ACK, $W$ is the time spent in the queue, $T_{0}$ is the value of the TCP timer and $p$ is the probability of loss.

In turn, this probability of loss $p$ depends on the arrival rate through the equation [6]

$$
p=1-\frac{\rho_{c}}{\rho}
$$


where

$$
\rho=\lambda \bar{X}
$$

where $\bar{X}$ is the mean service time of one TCP packet and

$$
\rho_{c}=\frac{\left(1-q_{0}-r_{0}\right) \bar{X}}{\left(q_{0}+r_{0}\right) \bar{X}+\left(\left(1-q_{0}-r_{0}\right) \bar{V}\right.}
$$

where $q_{0}$ and $r_{0}$ denote, respectively, the probability of having 0 packets in the queue after vacation and after service and are determined in a cross-layer fashion from the MAC layer. $\bar{V}$ is the mean vacation time which, for statistically identical users, is given by [7]

$$
\bar{V}=\left(n^{d}-1\right) \bar{X}
$$

where $n^{d}$ is the number of users in the system and is determined, in a cross-layer manner, from the flow level.

The time spent in the queue $W$ is given by

$$
W=\frac{\frac{1}{\lambda D} \sum_{j=1}^{K-1} j r_{j}+K p}{\lambda(1-p)}
$$

where $r_{j}$ is the probability of having $j$ packets in the queue after a vacation and $D$ is given by

$$
D=\left(q_{0}+r_{0}\right) \bar{V}+\left(1-q_{0}-r_{0}\right) \bar{X}
$$

As of the probability of a spurious time-out, it is given by

$$
\operatorname{Prob}\left(W+R T T>T_{0}\right)
$$

\section{NUMERICAL APPLICATION}

To keep the numerical application tractable, let users belong to one of three classes of distances depending on their distance from the base stations, close (class 1), far (class 3) and in between (class 2), with $d=100,200$ and $300 \mathrm{~m}$, respectively [8] and radio conditions, given by different values of $\Omega$ in Eqns. (1), (2) and (3) to users of class 3, 2 and 1, respectively. Let the total shared capacity $C_{s h}$ be equal to $1 \mathrm{Mbps}$. Although this value is not large, to ease the numerical application, we shall investigate the system performance at this scale, in terms of arrival rates and maximum numbers of users too. An upper scaling is straightforward.

Let elastic flows have an arrival rate equal to 0.2 and streaming flows have an arrival rate that starts at 0.01 and which is incremented by 0.02 . Without loss of generality, let the elastic users belong to one class only.

Let us first investigate the performance of PFS at the MAC layer. The average throughput under PFS (for $\tau=3$ ) is as plotted in Figure 3.

We can observe a higher throughput is achieved for user of class 1 over the one of class 2 who in turn has better performance over user of class 3. Even though PFS is fair, a user cannot be made to achieve a throughput higher than what its distance and fading conditions permit. Fairness is to be understood in terms of time opportunities to transmit rather than rate of transmission. In all cases, these users achieve

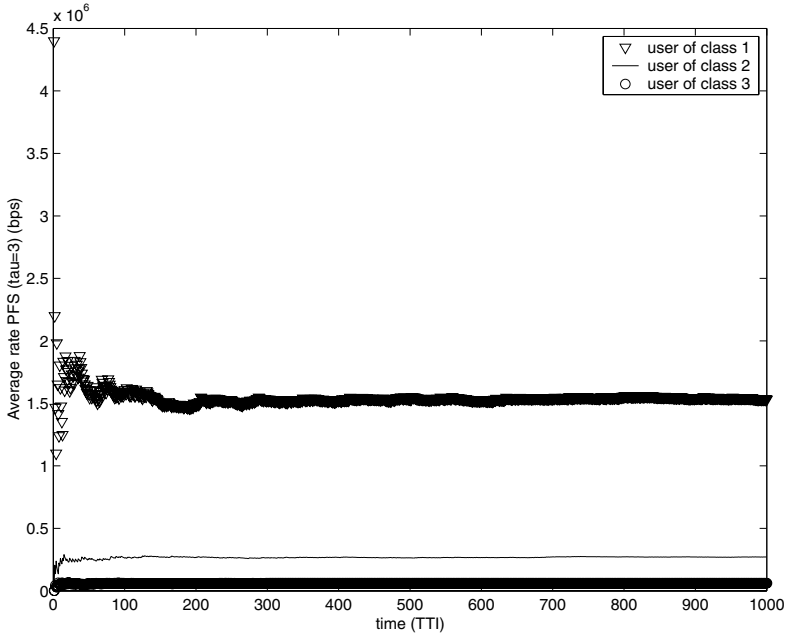

Fig. 3. Average throughput - PFS - $\tau=3$

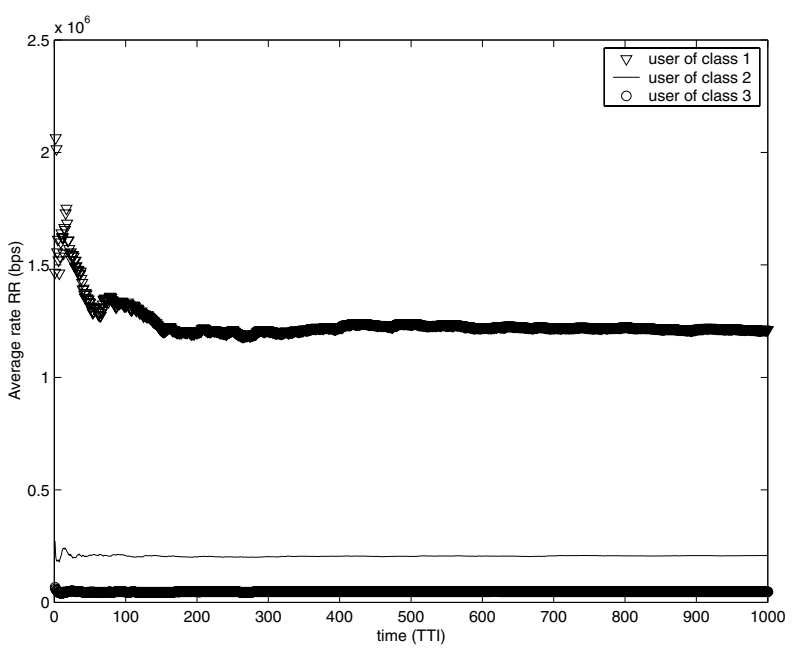

Fig. 4. Average throughput - RR

higher rates with PFS than with Round Robin (RR) (Figure 4). Max $\mathrm{C}$ over I, the algorithm that gives resources to the user experiencing the best radio conditions, makes user of class 1 obtain a much better throughput at the prohibitive cost of zero throughput for other users of the system (see Figure 5).

Now, we turn to the flow level. Figure 6 shows the mean transfer time of elastic users for different values of voice arrival rates, both analytically using the quasi-stationary approximation and numerically. We can observe a very good match between the result obtained making the quasi-stationary hypothesis and the exact one. The small values for the mean transfer argue further for such an assumption.

Figures 7 and 8 show, respectively, the blocking probability of streaming and data users for different values of voice arrival rates, both analytically using the quasi-stationary approximation and numerically. 


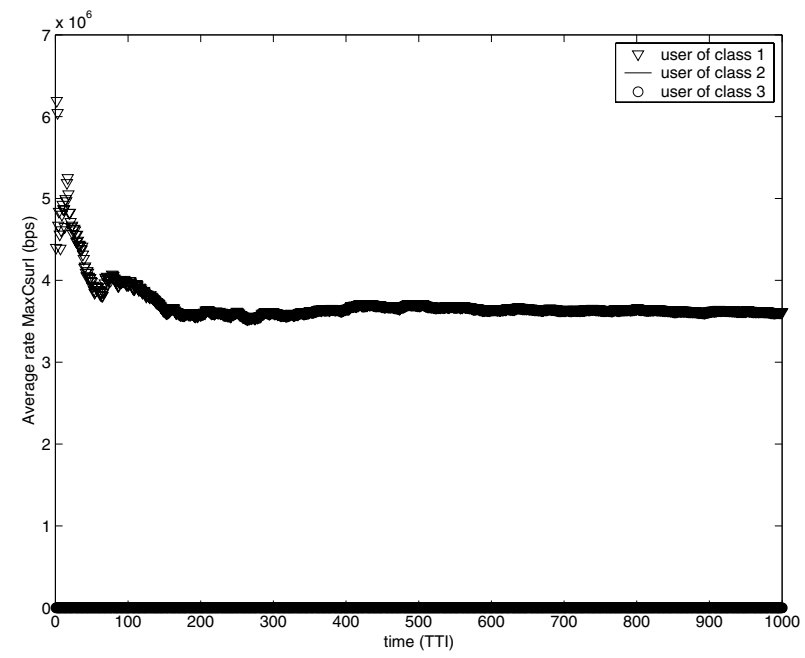

Fig. 5. Average throughput - Max C/I

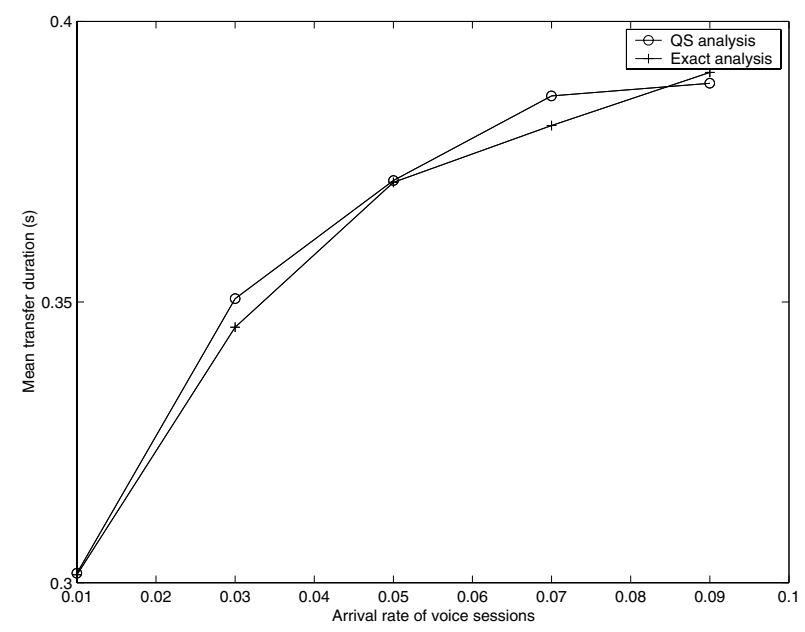

Fig. 6. Mean transfer time

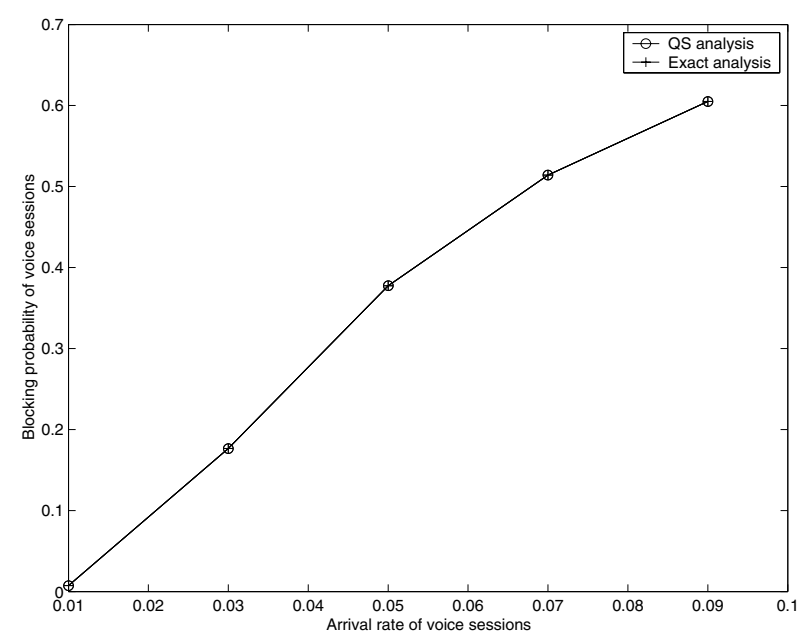

Fig. 7. Blocking probability for voice flows

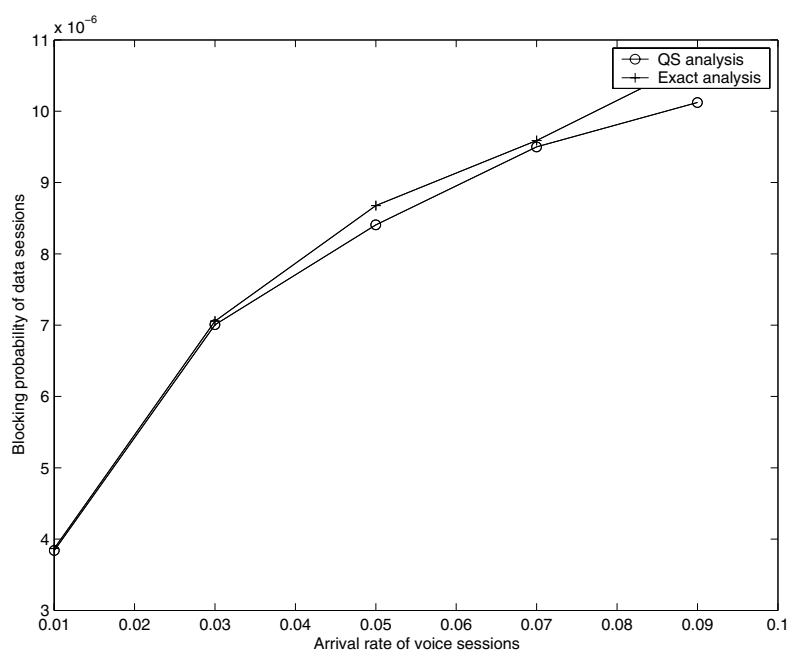

Fig. 8. Blocking probability for data flows

\section{CONCLUDING REMARKS}

In this work, we investigated the integration of streaming and TCP-based elastic flows in UMTS networks in relationship with opportunistic scheduling at the MAC layer as implemented in HSDPA as well as with TCP dynamics for data flows at the packet level. At the latter scale, TCP performs well in general, even over wireless links. When things go wrong however, the degradation may be really severe causing spurious time-outs and lesser throughput performance. In this case, PFS should be coupled with TCP dynamics as shown in [7] where a correction term is added to the $\frac{r}{R}$ ratio when making a scheduling decision.

Our next steps are on the UMTS/HSDPA capacity region by coupling the uplink and downlink since TCP flows in the downlink trigger an ACK flow in the uplink; and the latter may be restrictive in this case. If so, some novel proposals suggest the use of new coding schemes to offer reliability for data flows in an open-loop fashion without the need for ACKs.

\section{REFERENCES}

[1] P. Viswanath, D. Tse and R. Laroia, Opportunistic Beamforming using Dumb Antennas, IEEE Transactions on Information Theory, Vol. 48, No. 6, pp. 1277-1294, June 2002.

[2] N. Benameur, S. Ben Fredj, F. Delcoigne, S. Oueslati-Boulahia and J.W. Roberts, Integrated Admission Control for Streaming and Elastic Traffic, QofIS 2001, Coimbra, September 2001.

[3] J. G. Proakis, Digital Communications, Fourth Edition, MacGraw-Hill, 2001.

[4] T. Bonald, A. Proutiere, Wireless downlink data channels : User performance and cell dimensionning, ACM Mobicom, 2003.

[5] J. Padhye, V. Firoiu, D. Towsley and J. Kurose, Modeling TCP Reno Performance: A Simple Model and its Empirical Validation, IEEE/ACM Transactions on Networking, April 2000.

[6] S. K. Bose, Introduction to Queuing Systems, Kluwer/Plenum Publishers, 2001.

[7] T.E. Klein, K. K. Leung, Z. Haito, Improved TCP performance in wireless IP networks through enhanced opportunistic scheduling algorithms, GLOBECOM '2004, Dec. 2004.

[8] S-E. Elayoubi, T. Chahed, B. Elsaghir, Insuring priorities via admission control in WCDMA mobile systems, ITC19, Beijing, August/September, 2005. 